ÉGYPTE monde arabe

\section{Égypte/Monde arabe}

33 | 1998

Travailleurs et industrie à l'heure des réformes

économiques

\title{
Pauvreté et paupérisation en milieu urbain. Une enquête préliminaire
}

\section{Hasanayn Kishk}

Traducteur : Samia Rizk

\section{(2) OpenEdition}

\section{Journals}

Édition électronique

URL : https://journals.openedition.org/ema/1577

DOI : 10.4000/ema. 1577

ISSN : 2090-7273

Éditeur

CEDEJ - Centre d'études et de documentation économiques juridiques et sociales

\section{Édition imprimée}

Date de publication : 30 juin 1998

Pagination : 81-112

ISSN : 1110-5097

\section{Référence électronique}

Hasanayn Kishk, «Pauvreté et paupérisation en milieu urbain. Une enquête préliminaire », Égypte/ Monde arabe [En ligne], 33 | 1998, mis en ligne le 08 juillet 2008, consulté le 07 juillet 2022. URL : http:// journals.openedition.org/ema/1577 ; DOI : https://doi.org/10.4000/ema.1577

Ce document a été généré automatiquement le 7 juillet 2022.

Tous droits réservés 


\title{
Pauvreté et paupérisation en milieu urbain. Une enquête préliminaire
}

\author{
Hasanayn Kishk \\ Traduction : Samia Rizk
}

\section{NOTE DE L'ÉDITEUR}

Le texte qui suit est une version réduite d'un compte rendu d'enquête présenté dans le cadre du colloque « Les effets de la politique de privatisation sur la situation des travailleurs et des pauvres des régions urbaines ", qui s'est tenu du 26 au 28 février 1997 au Centre d'aide syndicale et ouvrière (Dar al-Khadamât al-Niqâbiyya wal-'ummâliyya) de Hilwân.

1 Les mesures relatives à la mise en place du programme «de stabilisation et d'ajustement structurel » n'ont fait qu'accentuer la détérioration de la situation économique et sociale des classes laborieuses en Égypte, tandis qu'on assiste à une redistribution du revenu et des biens en faveur des détenteurs de capitaux et au détriment des salariés. La baisse de la part du facteur humain dans le revenu national est due au gel des salaires, à la hausse des prix, à la suppression de nombreuses subventions, à l'augmentation des tarifs des services publics et des taxes indirectes, ainsi qu'à la suspension des recrutements dans la fonction publique et dans le secteur public, et à la mise à pied de la main-d'œuvre excédentaire. En revanche, les revenus fonciers augmentent avec l'élévation des taux d'intérêt, la réduction de l'impôt sur les gros revenus, l'exemption des taxes dont bénéficient les investisseurs privés et la réforme des relations entre les propriétaires fonciers et les locataires, etc. (Zakî, 1993a, p. 22) ${ }^{1}$

2 La pauvreté se répand davantage en milieu urbain. La proportion des ménages pauvres (juste au niveau du seuil de pauvreté ou au-dessous) qui était, en 1981, de 30,4\% dans les villes, est passée en 1990-1991 à $49 \%$ (Korayem, 1994)². La comparaison entre la pauvreté et la richesse permet de constater que l'une et l'autre connaissent une plus 
forte concentration dans les régions urbaines. Les $10 \%$ les plus pauvres de la population ne bénéficient que de $2,5 \%$ du total des revenus des familles vivant en milieu urbain, alors que la part des $10 \%$ les plus riches atteint 32,6 \% de ce total (soit 13 fois plus) ; en $1981-1982$, la part des uns et des autres était respectivement de 2,6\% et $25,9 \%$. Le décile supérieur voit sa part de revenu augmenter de $26 \%$ de l'ensemble du revenu national à $32,5 \%$ au détriment du reste de la population, chacun des déciles restants voyant sa part du revenu diminuer (Korayem, 1994, p. 39-41). Le niveau de pauvreté dans la partie urbaine des gouvernorats est élevé: les familles pauvres dépassent $50 \%$ de l'ensemble des ménages dans 13 gouvernorats (sur un total de 21 gouvernorats) et atteignent même $78 \%$ dans un cas. Il est plus bas à Damiette et PortSa'îd, modérément élevé au Caire, à Daqahliyya, Sharqiyya et Ismâ'îliyya, et très élevé à Buhayra, Asyût, Sûhâg et Aswân. Au Caire, 35,4\% des ménages vivent au seuil de pauvreté ou au-dessous. Cette proportion est de $76,1 \%$ à Buhayra, 77,6\% à Aswân, 76,9 \% à Asyût, 74,8 \% à Sûhâg, 47,5 \% à Gîza et 59,9 \% à Qinâ (Korayem, 1994, p. 29-30).

Le présent texte vise à présenter les premiers résultats d'une enquête réalisée au printemps 1996 sur ce thème.

\section{Cadre théorique et méthodologique}

\section{Définition des concepts}

\section{La pauvreté}

Trois types de définition sont souvent utilisés :

1. Une définition " objective " s'attache à fixer un « seuil de pauvreté », c'est-à-dire le revenu minimum nécessaire à l'individu ou au ménage pour faire face aux principales dépenses alimentaires et autres. Les individus qui vivent à ce niveau ou au-dessous font partie de la catégorie des « pauvres».

2. Une définition «subjective » considère la pauvreté du point de vue de l'individu lui-même ; s'il n'arrive pas à satisfaire ce qu'il juge être ses besoins essentiels, il se considérera comme pauvre.

3. Une définition «sociologique " considère comme pauvres ceux qui bénéficient d'une aide sociale et sont ainsi considérés comme étant pauvres par la « société » elle-même (Korayem, 1992, p. 4-5).

5 La plupart des études caractérisent les régions pauvres comme ayant une population composée de migrants ruraux, où les logements sont construits sans autorisation, les rues étroites, l'infrastructure et les services insuffisants (écoles, hôpitaux, terrains de jeux, etc.) (Korayem, 1992, p. 33). Par ailleurs, la population pauvre en Égypte se caractérise, selon ces études, par la malnutrition, les ménages nombreux (le père a un emploi d'appoint en plus de son emploi principal, les enfants travaillent très jeunes à l'intérieur ou à l'extérieur du foyer et prennent en charge les parents âgés), l'insuffisance des biens que possède le ménage, qu'il s'agisse de biens matériels ou de capital culturel.

6 Karima Korayem opte pour une définition de la pauvreté fondée sur les besoins fondamentaux ; c'est une définition qui permet de mesurer la pauvreté de façon plus globale que les définitions précédentes mais qui présente néanmoins des inconvénients, au premier chef desquels l'arbitraire avec lequel sont déterminés les 
besoins fondamentaux du ménage en produits alimentaires et non alimentaires, ainsi que les prix sur lesquels sont calculées les dépenses d'un ménage pour l'achat de ces produits, ces derniers connaissant une variation régionale. Ceci dépend en fait de plusieurs facteurs tels que le sexe, l'âge, la stature, le poids de l'individu et le type d'activité qu'il exerce (Korayem, 1992, p.35-37). Elle définit en outre un seuil de " pauvreté absolue » ou d'« extrême pauvreté ", qui correspond à un niveau inférieur à celui du seuil de pauvreté, mais supérieur à la limite en deçà de laquelle l'individu serait exposé à la famine. Ce revenu est calculé sur la base des dépenses minimales consacrées par un ménage aux produits alimentaires pour assurer à ses membres une alimentation correcte, telle qu'établie pour les ménages dans les régions urbaines et rurales (Korayem, 1994, p. 2).

7 Cependant, ces différentes définitions de la pauvreté la présentent comme s'il s'agissait d'une réalité naturelle. La plupart d'entre elles relèvent en fait de théories qui ramènent la pauvreté à la nature des choses et des êtres, c'est-à-dire à des différences biologiques, ethniques ou culturelles entre les individus ou les peuples. Elles occultent par conséquent le fait que la pauvreté est le produit de l'exploitation et de la spoliation exercées par les classes dominantes intérieures et étrangères, ainsi que l'origine historique de la pauvreté et son lien avec la naissance de la propriété individuelle des moyens de production. C'est pourquoi nous avons préféré, dans la présente étude, retenir le concept de paupérisation sans nier pour autant le profit que nous avons tiré des précieuses contributions de Karima Korayem concernant la mesure quantitative de la pauvreté et du seuil de pauvreté.

\section{La paupérisation}

8 La conjonction de l'exploitation étrangère (inégalité des échanges entre les pays capitalistes du centre et les pays de la périphérie, détérioration des conditions commerciales au profit des premiers, aggravation de la crise de l'endettement et du poids de la dette) et de l'exploitation intérieure (celle exercée par les classes capitalistes locales) a eu pour effet la paupérisation des producteurs directs (les travailleurs de l'industrie, de l'agriculture et des services), des petits producteurs des campagnes et des villes, et des catégories inférieures des couches moyennes (Korayem, 1994, p. 17). On distingue deux types de paupérisation :

1. La paupérisation absolue découle de la séparation des producteurs directs de leurs moyens de production. Cette séparation a donné naissance, dès le XIX ${ }^{e}$ siècle, à la classe des grands propriétaires fonciers, d'un côté, et, de l'autre, aux paysans démunis, dépossédés de leurs terres - soit par spoliation directe, soit par abandon collectif des villages, conséquence de l'imposition de la corvée, de la conscription forcée, des impôts et de l'endettement - qui sont les pères de la classe ouvrière égyptienne (Kishk, 1995, p.13-18). Cette situation s'est prolongée du fait de l'exploitation capitaliste qui, ruinant les petits agriculteurs, les a dépossédés de leurs biens, au profit d'une minorité de riches agriculteurs. Le processus de paupérisation absolue se poursuit, premièrement, à travers le marché des moyens de production où les petits agriculteurs achètent très cher (en raison du nombre d'intermédiaires) ce dont ils ont besoin; deuxièmement à travers le marché des produits agricoles qui sont vendus à un prix inférieur à leur valeur réelle ; et troisièmement à travers le marché du crédit dont les conditions de financement ne leur sont pas favorables (Kishk, 1995, p. 13-18). La persistance de cette séparation a entraîné une augmentation du taux de chômage dans les villes et les campagnes, contraignant les ouvriers à accepter des salaires équivalents au coût minimal de la reproduction de leur force de travail. En outre, le 
processus de paupérisation englobe le phénomène de l'emploi des enfants et des femmes pour un salaire inférieur à celui des hommes (Al-'Amrûsî, 1992) 3 .

2. La paupérisation relative est la réduction de la part relative des salaires dans le produit net généré par le travail; elle est due à l'augmentation du taux de la plus-value qui est une réponse $\mathrm{du}$ capital à la baisse du taux du profit moyen. La paupérisation conduit à la diminution du salaire réel face à l'accroissement des coûts des biens nécessaires à la reproduction de la force de travail : alimentation, habillement, santé, éducation, logement, etc.

\section{Le ménage}

9 Le mot ménage désigne, dans la présente étude, l'ensemble des personnes vivant en commun, au sein d'un même logement (même s'il s'agit de plusieurs étages), et prenant leurs repas en commun. Le ménage peut être constitué d'une famille restreinte (le noyau) qui comprend le couple - ou l'un des deux conjoints - et ses enfants, ou d'une famille élargie qui comprend plus d'un noyau. Les membres émigrés, à l'extérieur ou à l'intérieur, ne font pas partie du ménage (s'ils ont un lieu de résidence différent).

\section{Le revenu}

10 Ce terme désigne ici l'ensemble des revenus générés par les membres du ménage : salaires, rémunérations, primes, bénéfices, retraites, rentes immobilières (telles que les baux de terrains agricoles par exemple), gain généré par diverses activités lucratives (celles des «familles productives « : couture, petit commerce, etc.), et aides sociales. L'indemnité de repas n'est pas considérée comme source de revenu puisque seuls les ouvriers des secteurs étatiques de production et des services sont concernés.

\section{Les journaliers}

11 Ce terme désigne ici les personnes qui travaillent, dans les régions urbaines, dans ce que les économistes appellent le «secteur informel » ou «non réglementé ». Ces deux dénominations ont été utilisées pour la première fois il y a vingt ans dans les études sur le développement, pour désigner, dans un premier temps, les pauvres des régions urbaines en particulier, ou ceux qui vivaient dans les quartiers pauvres et surpeuplés, dans des logements extrêmement modestes construits sur des terrains qu'ils se sont appropriés sans droit, ainsi que ceux qui exercent des professions modestes telles que balayeurs, éboueurs, cireurs de chaussures, etc. Dans certains cas, ce terme désignait ceux qui exerçaient une activité de faible productivité et/ou à bas salaire, ainsi que les ruraux venus s'installer dans les villes puisque la plupart d'entre eux ne réussissaient pas à trouver un emploi dans le secteur formel. Ce secteur se caractérise par :

1. une certaine facilité à accéder au marché du travail et à en sortir : ce sont des activités qui, pour démarrer, ne nécessitent ni un capital important, ni de grandes aptitudes, ni beaucoup d'expérience ;

2. l'absence de protection étatique ou légale quant au niveau des salaires et aux conditions de travail (horaires, droits à un congé, assurances, âge des travailleurs, etc.) ;

3. les activités exercées, qui sont le plus souvent des activités commerciales (plus particulièrement dans le commerce de détail, la restauration et l'hôtellerie), ou de type artisanal dans les ateliers de menuiserie, les forges, les garages, dans l'industrie alimentaire ou encore de service auprès des particuliers (domestiques, cuisiniers, chauffeurs, etc.) ; 
4. le caractère irrégulier et instable, au jour le jour, de l'emploi.

\section{Objectif de l'enquête}

12 L'enquête visait à caractériser la situation économique et sociale de quelques types de pauvres des régions urbaines d'Égypte. Ces types recouvrent des échantillons sélectionnés parmi des ouvriers du secteur public industriel, des petits employés du secteur tertiaire, des journaliers et des chômeurs. Elle visait également à répertorier les changements survenus dans ces situations et, par conséquent, à répondre aux questions suivantes :

1. quelles sont les conditions d'existence des catégories les plus pauvres des régions urbaines?

2. Quel est l'impact de la politique d'ajustement structurel sur leurs conditions de vie? (Changements survenus dans la structure des dépenses.)

3. Quels sont les moyens utilisés pour assurer la reproduction des conditions d'existence?

4. Quelle perception les enquêtés ont-ils de leurs problèmes familiaux, des problèmes de leur quartier et du rôle des conseils locaux?

\section{Méthodologie}

13 Afin de répondre à ces questions, une enquête par entretien a été réalisée. auprès d'un échantillon représentatif. Le guide d'entretien comporte plusieurs axes portant sur les conditions d'existence des enquêtés, les changements qu'elles ont connus au cours des cinq dernières années et les formes de reproduction de cette existence.

\section{L'échantillon : principes de sélection et caractéristiques}

\section{Principes de sélection}

L'enquête globale porte sur un échantillon de 1000 individus. L'enquête préliminaire, dont nous présentons ici les résultats, concernait 100 personnes choisies dans les régions urbaines du Grand-Caire et dans des villes de trois autres gouvernorats : Gîza, al-Mahalla al-Kubra et Kafr al-Dawwâr, parmi les catégories les plus représentatives possible de la diversité réelle de la situation des pauvres en Égypte : ouvriers du secteur public industriel, employés du secteur tertiaire, journaliers et chômeurs.

15 Les individus interrogés ont été sélectionnés en fonction des variables suivantes : le sexe, l'âge, l'état matrimonial, le niveau d'instruction, le secteur d'activité, la position dans le ménage et le nombre d'individus que comporte ce dernier. Seuls 67 questionnaires ont été finalement complétés. Dans la suite du texte, le terme « échantillon » désigne ces 67 enquêtés.

\section{Caractéristiques de l'échantillon}

16 - Les hommes représentent environ $63 \%$ des enquêtés et les femmes $37 \%$. La tranche d'âge des 20-39 ans représente environ les trois quarts des enquêtés et le quart restant est pris dans la tranche d'âge 40-59 ans (voir tableau 1). 
17 - Environ $30 \%$ des enquêtés sont des ouvriers du secteur public industriel; une proportion comparable travaille dans le secteur tertiaire public ; $13 \%$ dans le secteur privé ; 17 \% sont des « journaliers » et $10 \%$ des chômeurs (voir tableau 2).

18 - $37 \%$ des enquêtés sont analphabètes : une petite moitié de ces derniers se retrouvent parmi les «journaliers » et les chômeurs ; la proportion des diplômés du secondaire est relativement élevée et atteint 32,8 \% des enquêtés ; 5,9\% sont diplômés d'un institut technique ( 2 ans après le certificat de fin d'études secondaires ; 1,5 \% sont diplômés d'un institut de formation professionnelle (cinq années d'études après le certificat de fin d'études préparatoires) et $3 \%$ détiennent un diplôme universitaire (voir tableau 3).

19 - La proportion des personnes mariées dépasse les deux tiers des enquêtés (70\%), celle des célibataires est d'environ $25 \%$; celle des veufs $2,9 \%$ et il y a une seule divorcée (voir tableau 4).

20 - Les ménages comptant entre 5 et 7 individus représentent $53 \%$ des ménages enquêtés ; ceux qui sont constitués de 1à 4 personnes représentent $35,8 \%$; et ceux de 8 à 12 personnes 10,5\% (voir tableau 5). Parmi ces ménages, on relève un nombre relativement important de familles nombreuses, ce qui semble traduire une tendance chez les « pauvres » des régions urbaines à avoir beaucoup d'enfants.

21 - 25 \% des enquêtés sont de Hilwân, 14,9 \% de Ma'sara, 13,4 \% de Imbâba, 5,9 \% de Bulâq al-Dakrûr et Mît 'Uqba, 7,5 \% de al-Saff, 19,9 \% de Mahalla al-Kubra, et 14,9 \% de Kafr alDawwâr.

\section{Nature des activités des enquêtés}

22 Dans le secteur public industriel, les professions exercées par les salariés de l'échantillon se répartissent de la manière suivante : un contrôleur de qualité à l'usine sidérurgique, cinq ouvriers de production dans la même entreprise, un soudeur à la société Al-Masriyya li-l-harâriyyât (industries du verre, de la céramique, etc.), un ouvrier d'affûtage à l'usine de tuyauterie al-Nasr, un inspecteur de qualité à l'usine militaire 99, un technicien de production à la société de coke, un chef des sections textiles à la Société Misr de filature de Kafr al-Dawwâr, un électricien de haute tension et une ouvrière de production de la même société, quatre ouvrières de production de la Société Misr defilature et de tissage à Mahalla al-Kubra, une ouvrière de production dans une société d'appareils électroniques (télévisions, etc.) et une ouvrière de production à la société de produits pharmaceutiques Memphis.

Dans le secteur tertiaire public, les professions exercées par les salariés de l'échantillon se répartissent de la manière suivante: un aide-électricien, un mécanicien de maintenance, un peintre au pistolet, un ouvrier de manutention, un plâtrier, un ouvrier de dépôt, un magasinier, un garde, un chef de service administratif, un comptable, une employée dans les travaux de secrétariat, une employée sans qualification précise, deux infirmières, un employé des postes, un représentant d'achat à l'Institut d'études supérieures en métallurgie al-Tibbîn, un enseignant d'école secondaire industrielle, une institutrice et deux agents administratifs femmes.

24 Dans le secteur privé, les professions exercées par les ouvriers de l'échantillon se répartissent de la manière suivante : une femme de ménage, une vendeuse dans une librairie, un boulanger, un charpentier, un tailleur de pierre, un tapissier automobile, 
un ouvrier des dépôts de bouteilles à gaz, un plâtrier qui est en même temps petit entrepreneur.

Les journaliers se répartissent entre : deux marchandes de légumes, un marchand de tapis faits main, un ouvrier maçon (porteur de pierres, de sable et autre matériel), un vendeur de cassettes et d'appareils électriques, un vendeur de fèves ambulant, un marchand de légumes, une épicière, un vendeur de fèves (dans une échoppe), une couturière, un ouvrier qui prépare les chutes de tissus pour les revendre aux fabricants de nattes tressées main. un emploi. Une autre, de 17 ans, a le certificat de fin d'études primaires, ne sait pas bien lire. Elle a travaillé comme vendeuse dans une petite boutique; elle ne trouve pas d'emploi car " les sociétés exigent un diplôme et les ateliers recrutent ceux qui ont de l'expérience ». Quant à la troisième chômeuse, elle a 27 ans, est mariée et analphabète.

\section{Premiers résultats}

\section{Revenu des ménages}

Le quart des enquêtés déclare un revenu mensuel inférieur à $200 \mathrm{Ee}^{5}$ et la moitié environ d'entre eux sont des journaliers. $45 \%$ des enquêtés touchent entre 200 et $400 £ e$, les deux tiers étant des ouvriers du secteur public industriel; $18 \%$ des enquêtés perçoivent entre 400 et $600 £$ ₹ $; 12 \%$ ont un revenu supérieur à 600 £e (voir tableau 6).

Sachant que le revenu annuel correspondant au seuil de pauvreté dans les régions urbaines d'Égypte, en 1990-1991, était évalué à 3 347,4 £e, soit une moyenne mensuelle de 298,9 €e (al-'îsawî, 1989, p. 101 -103), et que ce revenu est calculé pour une famille urbaine moyenne de 4,6 individus, et si nous tenons compte de la tendance à la hausse des prix durant le premier semestre 1990, des produits alimentaires et non alimentaires correspondant aux besoins fondamentaux, on peut facilement en déduire que près de la

Égypte/Monde arabe, 33 | 1998 
moitié des ménages touchés par l'enquête vivent au niveau du seuil de pauvreté ou audessous.

\section{Dépenses alimentaires}

31 Selon l'étude de Karima Korayem (1994, p. 1), les dépenses alimentaires s'élevaient à $49,6 \%$ du revenu des ménages pauvres en 1991. Notre enquête révèle que $65 \%$ des personnes interrogées y consacrent plus de $40 \%$, et parmi elles, $20 \%$ y consacrent entre 60 et $80 \%$, et environ $27 \%$ entre 80 et $100 \%{ }^{6}$ (tableau 7 ).

Le repas dispensé sur le lieu de travail apporte une amélioration relative à l'alimentation des travailleurs, mais seuls ceux du secteur public bénéficient de cet avantage. Pour les ouvriers du secteur industriel, il s'agit soit d'un repas froid, soit d'une indemnité de repas, soit d'une compensation en nature dont la composition diffère d'une usine à l'autre : dans l'une des usines c'est une boîte de margarine (deux kilos), un kilo de lentilles, un kilo de riz, un kilo d'huile, trois petits paquets de thé, le tout équivalant à $30 \mathrm{Ee}$. Dans d'autres usines, cette compensation est constituée d'une boîte de margarine (deux kilos), un kilo de sucre, un kilo de riz, trois kilos de lentilles, cinq savons, cent grammes de thé, une boîte de halâwa (600 grammes) plus 6 £e et d'autres espèces de compensations en nature. Quant à l'indemnité de repas mensuelle, elle varie entre 12 et 16 £e pour les ouvriers du secteur industriel, et entre 4 et 12 fe dans le secteur tertiaire. Dans l'une des usines où elle atteignait 24 £e, elle a été réduite à $12 \mathrm{fe}$ avant d'être supprimée complètement. Les ouvriers du secteur industriel préfèrent en général la compensation en nature à l'indemnité de repas; ils estiment que "ça aide " et que c'est plus "utile». Un des travailleurs du secteur tertiaire se plaint de la somme reçue et se demande à quoi peut bien servir la livre qui lui est versée ${ }^{7}$.

\section{Niveau d'instruction des enfants des enquêtés}

$53,4 \%$ des enfants des enquêtés sont scolarisés à différents niveaux du système éducatif: la moitié d'entre eux sont dans le cycle primaire, le quart dans le cycle préparatoire, environ un cinquième dans le secondaire ou dans l'enseignement technique, et seulement $5 \%$ dans l'enseignement universitaire (quatre cas). $10 \%$ des enfants se sont arrêtés après avoir achevé un cycle d'études : la moitié ont été jusqu'au certificat de fin d'études secondaires, mais près d'un quart se sont contentés du certificat de fin d'études préparatoires. $14 \%$ des enfants ont abandonné leurs études en cours de route, à différents niveaux, principalement au cours du cycle préparatoire (voir tableau 8). La proportion de ceux qui s'arrêtent avant le certificat de fin d'études secondaires est donc relativement élevée (plus de la moitié), ce qui signifie que l'analphabétisme tend à se reproduire. Ces pourcentages sont les plus importants parmi les enfants de journaliers. Nous analyserons ces résultats lorsque nous parlerons des formes de reproduction de l'existence sociale des enquêtes, parce que la détérioration des conditions socio-économiques des pauvres pousse les enfants à entrer plus tôt sur le marché du travail afin de ramener un revenu supplémentaire. S'ajoute à cela le coût élevé de l'enseignement en général, et de l'enseignement universitaire en particulier. 


\section{Dépenses d'éducation et de santé} uniforme, cours particuliers et groupes de soutien). De nombreuses familles ont recours aux services offerts par les mosquées, les églises et certaines associations privées en matière d'enseignement. Les résultats de l'enquête montrent que près d'un quart des ménages consacrent à l'éducation plus de $20 \%$ de leur revenu (environ $12 \%$ y consacrent plus de $40 \%) ; 30 \%$ y consacrent entre 10 et $20 \%$, seulement $22 \%$ y consacrent moins de $10 \%$ (tableau 9). Si la majorité des enquêtés (63\%) consacrent moins de $10 \%$ du revenu du ménage aux soins de santé, ils sont $30 \%$ à y consacrer plus de $20 \%$ et $7 \%$ à y consacrer plus de $30 \%$ (tableau 10). Dans certaines régions où se trouvent les ouvriers du secteur public industriel, telles que al-Ma'sara, Hilwân et alTibbîn, les maladies pulmonaires, les allergies et les maladies hépatiques sont très répandues. En outre, la plupart des enquêtés et leurs familles souffrent de maladies cardiorespiratoires, ophtalmiques et osseuses. Ceci s'expliquerait par les multiples sources de pollution dans ces régions.

\section{État du logement}

La grande majorité des ménages sont locataires : 49,3\% vivent dans des appartements de location (dont 14,9\% dans des appartements loués auprès de l'employeur), 5,9\% habitent une chambre de location avec sanitaires communs, 2,9\% louent une maison. $23,9 \%$ vivent dans une maison dont le père de l'enquêté est propriétaire, $13,5 \%$ sont propriétaires de la maison où ils vivent, et $2,9 \%$ de leur appartement. Certains logements d'ouvriers se caractérisent par leur surface limitée, l'absence de cuisine, de tout-à-l'égout. Ils sont mal aérés, très modestement meublés, et certains sont sans électricité. La plupart de ces logements ne sont équipés que du strict minimum en matière d'électroménager. L'un des logements, dont l'enquêté est propriétaire, est constitué d'une pièce unique, vide, dont la toiture est constituée de planches de bois et de chaume ; le sol est en ciment et il n'y a pas d'électricité.

Quant aux travailleurs du secteur tertiaire public et du privé, ils habitent, pour certains, dans des quartiers informels où les conditions d'hygiène laissent beaucoup à désirer : remontée des nappes d'eaux souterraines, absence d'égouts, amoncellement des ordures, etc. Les rues sont étroites et non aménagées, et l'électricité n'y a pas encore été introduite (comme dans le quartier d'al-Munîra al-gharbiyya à Imbâba). La moitié de ces logements présentent les mêmes caractéristiques que ceux des ouvriers de production.

journaliers et chômeurs habitent pour la plupart des logements exigus et insalubres. Certains se trouvent dans des quartiers informels sans égouts ni eau courante où les insectes pullulent. Mais la plupart de ces logements sont équipés des principaux appareils électroménagers : réfrigérateur, gazinière, machine à laver et téléviseur.

\section{Évolution des revenus et des dépenses des ménages}

Le guide d'entretien comportait des questions sur les changements survenus dans le revenu et les dépenses du ménage depuis le début des réformes économiques. Dans la 
plupart des cas, le revenu du ménage a augmenté dans la dernière période (notamment celui du chef de ménage homme, ou femme, si cette dernière exerce une activité rémunérée). Cette augmentation n'est cependant pas en rapport avec l'augmentation du coût de la vie.

\section{Dépenses alimentaires}

Il ressort de l'enquête que les ménages des ouvriers industriels (au nombre de 9) ont été contraints de diminuer leur consommation de viande tant sur le plan de la quantité que de la fréquence. Pour d'autres, cette réduction s'étend à la consommation de volaille et de poisson. La plupart de ces ménages se contentent des fruits et des légumes les moins chers et de certains féculents. Certains n'ont pas eu à modifier leur mode de consommation, car, depuis de longues années, leur budget est tellement modeste qu'ils ne font pas partie des catégories sociales qui consomment ces produits alimentaires. L'un de ces ouvriers, dont la famille est constituée de six membres et dispose d'un revenu de $525 \mathrm{fe}$, affirme que, pour lui, « rien n'a changé ; j'emprunte parfois à la caisse de solidarité ». Une ouvrière mariée dit que "la différence est minime, rien n'a changé ». Elle fait vivre une famille de trois personnes avec un revenu mensuel de $175 £$ e.

Parmi les travailleurs du secteur tertiaire public, on note les mêmes changements : réduction de la consommation de viande, de poisson, de fruits et de certains féculents, plus grande consommation des légumes peu chers, ainsi que de pain, de riz et de pâtes. De même, chez les ouvriers du secteur privé, deux familles de six membres chacune ont été réduites à se passer complètement de viande ; la première a un revenu mensuel de $300 £$ e, la seconde de $340 £$. La plupart des ménages couverts par l'enquête consomment davantage d'aliments qui « remplissent le ventre » indépendamment de leur valeur nutritive (légumes farcis, kusharî et pâtes).

\section{Dépenses non alimentaires}

41 Celles-ci comprennent les dépenses d'éducation, de santé, d'habillement, de loisirs, etc. Nous avons déjà évoqué les difficultés que rencontrent la plupart des ménages dues à l'augmentation des coûts de l'éducation et des soins de santé, et le fait que, dans la plupart des cas, les enfants ne sont pas scolarisés ou ne poursuivent pas leurs études; ils sont obligés de travailler pour augmenter le revenu et pouvoir faire face à la hausse des prix. Concernant les dépenses d'habillement, les données recueillies sont insuffisantes pour se prononcer. Nous savons cependant que certains ménages achètent le minimum en matière d'habillement (un vêtement d'hiver et un autre d'été) en participant à une gam'iyya ${ }^{8}$, ou en puisant dans les "primes de rendement » que perçoivent les ouvriers du secteur public, ou encore en achetant à. crédit à la gam'iyya istihlâkiyy $a^{9}$ ou encore grâce à l'aide d'associations caritatives qu'ils reçoivent lors des fêtes (un seul cas) ou aux cadeaux envoyés par des membres de la famille qui travaillent dans les pays arabes. Seul un petit nombre de ménages se rendent dans les jardins publics à l'occasion de Shamm al-Nasîm (fête du printemps traditionnellement célébrée par tous les Égyptiens), et un nombre plus réduit encore de ménages vont à la mer lors des vacances d'été. 


\section{Les moyens de préservation de l'existence}

42 La préservation et la reproduction de l'existence des pauvres des régions urbaines revêtent plusieurs formes: la constitution de familles nombreuses, le travail de la femme, la migration interne et externe, la création d'associations ou d'organisations regroupant des personnes originaires d'une même région (baladiyya), l'utilisation des services offerts par les mosquées et les églises ainsi que des dons de bienfaisance sont quelques-unes de ces formes.

\section{La constitution d'une famille nombreuse} sont très vite sur le marché du travail et rapportent un complément de revenu au ménage. Dans l'une des familles d'ouvriers du secteur public industriel, deux enfants ont abandonné l'école avant d'avoir terminé le cycle primaire. Ils travaillent dans un atelier. Dans une autre famille, le fils a abandonné l'école dès le cycle primaire et travaille comme boulanger. Parmi les travailleurs du secteur tertiaire public, l'un des fils (diplômé du secondaire technique) travaille parfois, quand l'occasion s'en présente, comme maçon ou comme peintre ; un autre travaille comme chauffeur (il a abandonné l'école à l'issue du cycle primaire). travaille comme vendeur dans une boutique de prêt-à-porter, un autre est vendeur dans un magasin de chaussures alors que le frère d'un autre enquêté est ouvrier agricole (sans instruction).

Chez les enfants des journaliers, on trouve un nombre relativement élevé d'analphabètes ou d'enfants qui, ayant abandonné l'école, travaillent dans le « secteur informel " (garçon de café, ouvriers dans le secteur du bâtiment, ou dans un petit restaurant que possède la famille, couturière, etc.). L'un d'eux travaille toutefois comme ingénieur et l'une des filles effectue un stage dans un cabinet d'avocats. 


\section{Le travail des femmes}

48

Le travail des femmes revêt plusieurs formes: emploi dans la fonction publique, activité rémunérée dans le secteur public ou privé, travail à la journée, travail à domicile (préparation de mukhallalât - condiments de fruits et de légumes - de confitures, broderie et couture, etc.). Le travail à domicile combine activités rémunérées permettant d'accroître le revenu familial, et travaux - notamment de couture - permettant de limiter les dépenses.

les femmes d'ouvriers, on trouve une employée dans la même société que son conjoint (sidérurgie), en disponibilité pour élever ses enfants depuis huit ans : elle dispose d'une machine à coudre qui lui permet de confectionner les habits de ses enfants, elle élève de la volaille sur son balcon, prépare des mukhallalât, de la confiture et du yoghourt: "tout cela permet de faire des économies par rapport aux prix du marché ». La femme d'un autre ouvrier du secteur public industriel est enseignante dans une école primaire, mais elle ne donne pas de cours particuliers. Une autre " prépare des mukhallalât et s'est acheté un four en tôle pour cuire le pain à domicile ». Une autre encore confectionne les habits de ses enfants et fait des mukhallalât. L'une des enquêtées travaille comme ouvrière dans une société de textiles et prépare des mukhallalât pour la famille. Une autre travaille dans une société de produits pharmaceutiques et fait de la couture pour la famille, mais aussi pour d'autres clients (contre rémunération).

50 Parmi les familles des enquêtés, on trouve une enseignante du primaire et une mère d'un des enquêtés qui confectionnent les habits de leurs enfants, y compris ceux qui sont mariés. L'épouse d'un autre confectionne les habits de ses enfants et fait de la couture (contre rémunération). Un des ouvriers qui possède un kiosque de cigarettes et de confiserie se fait aider par son épouse et ses filles. L'épouse d'un aide électricien dans une entreprise publique confectionne les habits de ses enfants et fait de la couture pour d'autres clients (contre rémunération). La mère d'un ouvrier confectionne les habits de ses petits-enfants, alors que son épouse fait du tricot.

1 Parmi les travailleurs du secteur privé, la femme de l'un d'eux confectionne les habits de ses enfants et fait du crochet (contre rémunération) mais il s'agit d'un petit revenu. Pour faire face au coût de la vie, une des enquêtées a dû faire des travaux de ménage (son époux est technicien de surface). Une autre vend « des radis et du gargîr (roquette) devant sa maison » et enfin, une troisième (veuve), des légumes.

\section{La migration interne et externe}

52 La plupart des pères des enquêtés avaient quitté la campagne pour la ville. À la génération suivante, la migration change de nature. Parmi les ménages couverts par l'enquête, on ne relève que deux cas de migration interne : un maçon a quitté la HauteÉgypte pour s'installer au Caire et un autre, dont la famille est installée au Caire, travaille comme gardien à Sharm al-Shaykh. On rencontre en revanche plusieurs cas d'émigration vers les pays arabes.

53 L'émigration est en effet un autre moyen de préservation et de reproduction de l'existence de ces ménages. Parmi les ménages d'enquêtés, le père d'un des ouvriers est parti pour de longs séjours dans les pays arabes; un des ouvriers est parti travailler en Irak en 1982 ; il y est resté un an et a réussi à économiser 3000 £e pour revenir se 
marier. Les deux frères d'un ouvrier sont partis, l'un en Arabie Saoudite pendant cinq ans "pour démarrer sa vie... il est rentré, s'est marié et habite à l'extérieur »; le deuxième est parti au Liban et " cela fait onze ans qu'on est sans nouvelles ». Ce même ouvrier dit, à propos des causes de l'émigration, que «le salaire est trop faible et ne permet pas d'envisager le mariage; comment payer un appartement ou couvrir les dépenses? Il faut partir dans n'importe quel pays.» Un autre ouvrier est parti en Libye ; il est rentré au bout de six mois. De même, l'époux d'une ouvrière est parti pour deux ans en Arabie Saoudite.

Dans les ménages des travailleurs du secteur tertiaire, l'époux d'une des enquêtées est parti en Libye pour six mois, le père d'une autre est parti en Irak et en Arabie Saoudite, et un enquêté est parti en Irak pour un an et il raconte « ... je suis parti parce qu'avec un salaire de 23 £e que pouvait-on faire en 1979 ? Je veux former une famille, fonder un foyer; le départ est une issue ». Un des enquêtés a fait plusieurs séjours en Libye (quatre années au total), et un autre a passé deux ans en Irak pour faire vivre sa famille.

Dans le secteur privé, un plâtrier est parti en Irak, en Libye et en Arabie Saoudite (sept années au total) et a pu construire une maison pour les siens ; un autre ouvrier est parti en Arabie Saoudite pour un an durant lequel il a réussi à économiser 2000 riais.

Un journalier parti en Irak pour un an a pu économiser $3000 £ e$; un autre a passé quatre ans en Libye; avec ses économies, il a pu couvrir les dépenses de ses enfants et leur construire une maison.

57 Ceux qui n'ont pas eu la possibilité de partir ne rêvent que de cela. Il y en a en effet qui n'ont même pas de quoi payer les papiers nécessaires et le visa du pays d'accueil.

\section{Le second emploi, ou la double journée de travail}

58 La détérioration de leur situation matérielle pousse certains à rechercher un salaire d'appoint en prenant un second emploi en plus de leur activité principale. Ce moyen de préservation de l'existence a pour effet de prolonger la journée de travail et, par là même, d'exposer la classe ouvrière à une plus forte exploitation.

59 À titre d'exemple, un travailleur du secteur tertiaire possède un kiosque de cigarettes et de confiserie qui lui rapporte $50 \mathrm{Ee}$ par mois pour «compenser le salaire insuffisant ». Un ouvrier de peinture au pistolet travaille tous les jeudis et vendredis comme peintre à la journée pour 10 à $15 £$ fe par jour. Un troisième a une petite épicerie qui lui rapporte $100 \mathrm{Ee}$ par mois; il se fait aider par ses enfants, surtout pendant les vacances d'été. Un autre arrive difficilement à concilier son emploi d'agent de sécurité dans un magasin du secteur public et le travail de maçon dix jours par mois avec une rémunération de 20 £e par jour; ça lui fait une moyenne de 16 heures de travail par jour « Je travaille comme agent de sécurité, un poste de nuit contre un salaire mensuel de $46 £ e . .$. On dit que l'employé ne doit pas travailler ailleurs, mais que peut faire un tel salaire?».

60 Un ouvrier du secteur public industriel résidant à al-Saff va régulièrement à la pêche ; c'est essentiellement grâce au fruit de cette dernière que sa famille se nourrit.

\section{Les dons de bienfaisance}

61 L'enquête montre que ces dons peuvent être soit individuels, versés par certaines personnes dans des buts précis, soit institutionnels comme ceux des associations qui 
offrent, à travers les mosquées et les églises, des services peu onéreux dans les domaines de la santé et de l'enseignement. Ainsi, une veuve dont les enfants sont scolarisés et qui possède une très petite épicerie reçoit, à l'occasion des fêtes, une aide en nature : tissus et viande, qui représente pour elle un soutien appréciable.

\section{La perception de la situation}

L'étude des formes de conscience ne faisait pas partie des objectifs de la présente recherche. Nous nous sommes donc limités à trois questions directement liées à notre objet à savoir, la perception qu'ont les enquêtés des principaux problèmes auxquels sont confrontées leurs familles, des problèmes des quartiers où ils vivent, et enfin du rôle des Conseils locaux.

\section{La perception des problèmes auxquels sont confrontées les famille}

63 Le problème que les familles ressentent le plus durement est celui de l'insuffisance des revenus et de la cherté de la vie. En effet, telle a été la réponse des trois quarts des ouvriers du secteur public industriel et tertiaire, de la totalité des travailleurs du secteur privé et de plus des deux tiers des journaliers (y compris les chômeurs). Le problème était parfois évoqué de manière indirecte : «Je suis malheureux de sentir que je n'arrive pas à satisfaire tous les besoins de mes enfants en nourriture et habillement", ou encore "le seul problème, c'est que nous sommes amenés à nous passer complètement des choses auxquelles nous étions habitués, ou à n'acheter que le strict minimum ", ou encore "le plus gros problème c'est la facture d'électricité et d'eau ainsi que les frais d'éducation qui peuvent être exigés à un moment inopportun, ce qui nous oblige à emprunter ", etc.

Vient en seconde position le problème de l'endettement : un tiers des travailleurs du secteur tertiaire public, la moitié des travailleurs du secteur privé et un cinquième des journaliers et chômeurs le mentionnent.

65 Viennent ensuite les problèmes conjugaux et l'état psychologique déplorable des conjoints en raison de leur revenu insuffisant, le problème du logement (espace réduit et insalubre) et enfin le chômage, l'absence d'égouts, les problèmes de l'éducation des enfants, la maladie du conjoint, les trop longues heures de travail et la pollution.

\section{Paupérisation et environnement : remarques}

66 La grande majorité des enquêtés s'accordent pour dire que leur famille souffre de la détérioration de leur environnement. La situation la plus pénible est celle de Ma'sara où les retombées des cheminées de l'usine de ciment, l'eau des égouts et le canal alKhashshâb forment un étang de moisissure propice aux insectes et aux bêtes nuisibles (moustiques, rats, serpents). Sur ses bords s'entassent les ordures qui, en brûlant, dégagent des fumées toxiques. Les habitants de cette région souffrent également des coupures d'eau. On retrouve la même situation à al-Tibbîn et surtout à al-Tibbîn alBalad dont l'air pollué est chargé d'une poussière noire (se dégageant de l'usine de briques) et d'une poudre de ciment (de l'usine Kafr al-'Uluw), et dont l'environnement est dégradé par la présence d'un canal, du trop-plein des égouts et des ordures. Quant au quartier de 'Arab Râshid à Hilwân, il est extrêmement sous-équipé, les égouts y débordent régulièrement, les ordures s'entassent dans les rues et les enquêtés se 
plaignent de l'insuffisance de l'alimentation en eau. Dans un quartier d'Imbâba (alMunîra al-gharbiyya), il n'y a pas d'eau courante et même les secteurs qui en ont endurent de fréquentes coupures qui durent plusieurs heures. Les habitants du quartier de Mît-'Uqba se plaignent du débordement des égouts et du bruit. À Kafr al-Dawwâr, toutes les familles des travailleurs de l'usine textile souffrent de la poussière de coton, cause de maladies pulmonaires, et aussi du débordement des égouts. À Mahalla alKubra, les enquêtés se plaignent des ordures entassées dans un des canaux et sur ses bords, du débordement des égouts et des moustiques.

La plupart des cas couverts par l'enquête estiment que le "Conseil local» dans ces quartiers ne joue aucun rôle concret dans la lutte contre la détérioration de l'environnement si ce n'est quelques efforts partiels consentis dans les régions de alMa'sara et de al-Tibbîn al-balad. Ces efforts se limitent au ramassage des ordures qui se trouvent dans le canal et sur ses bords, mais, précisent les enquêtés, on ne s'en débarrasse pas complètement. Certains enquêtés de ces deux mêmes quartiers évoquent le fait que le suintement des réservoirs des égouts atteint un tel niveau qu'il arrive jusqu'à 25 centimètres de l'entrée des maisons, provoquant des maladies dans les familles.

Tout ce qui précède correspond, d'une manière générale, à la détérioration des conditions de l'environnement dans les quartiers pauvres, ou à ce que l'on appelle actuellement « les quartiers informels ». Dans ces quartiers, les problèmes sociaux dus à la paupérisation (malnutrition, santé, éducation et logement) s'accumulent et, l'absence, la rareté ou la décadence des services publics engendrent d'autres problèmes environnementaux qui interagissent avec les premiers. Le retrait progressif de l'État (liquidation du secteur économique étatique, compression des dépenses publiques sur les services et libération des forces du marché de toutes restrictions) a et aura pour effet une paupérisation plus intense. Cela provoquera en même temps la détérioration du milieu (urbain ou rural) dans lequel vivent les pauvres, ce qui signifie que les problèmes de la pauvreté et ceux du milieu constituent les deux faces d'une même médaille.

\section{La perception des problèmes de la zone de résidence}

Les problèmes relatifs à la détérioration des services (l'absence de tout-à-l'égout ou le débordement des égouts ainsi que les coupures d'eau et d'électricité) occupent la première place dans les problèmes de quartier évoqués. Plus du tiers des enquêtés se plaignent du non-raccordement de leur quartier au tout-à-l'égout ou du débordement des égouts, un quart des coupures d'eau ou de l'absence d'eau potable, et le reste de fréquentes pannes d'électricité, voire de l'absence d'électricité dans certains quartiers habités.

70 Le problème de la pollution vient juste après, qu'elle soit due au ciment, aux gaz que dégagent les usines ou à l'accumulation des ordures dans les rues et à la prolifération des insectes (mouches et moustiques). Viennent ensuite les problèmes découlant de l'absence d'hôpitaux et de services sanitaires ainsi que le manque de coopération entre les gens. Les problèmes de la pauvreté et du chômage sont souvent vus comme des facteurs expliquant le délabrement du quartier. La délinquance des jeunes (rixes où sont utilisés des couteaux et parfois des sabres) est fréquemment mentionnée. 


\section{L'opinion sur le rôle du Conseil local}

71 Une majorité d'enquêtés estime que le Conseil local ne joue aucun rôle ou même n'en a tout simplement jamais entendu parler : les salariés du secteur public sont plutôt dans le premier cas, ceux du privé ainsi que les journaliers et chômeurs sont plus nombreux dans le second cas. Certains ont tout de même mentionné les quelques services présentés par ce conseil (eau, électricité, début des travaux de construction du tout-àl'égout et leur utilité pour le quartier). D'autres pensent que le conseil n'est utile qu'à ceux qui en font partie.

72 Il apparaît donc de ce qui précède que la conscience aiguë des problèmes rencontrés par les familles et le quartier, à savoir le revenu insuffisant, le coût élevé de la vie, le chômage, la pollution et la détérioration des services, ne s'accompagne d'aucune attente à l'égard du Conseil local, considéré comme ne contribuant nullement à l'amélioration des conditions des habitants.

\section{Conclusion}

Les pauvres des régions urbaines d'Égypte couverts par l'enquête souffrent à l'évidence d'une paupérisation croissante. Les principaux résultats de l'enquête peuvent être résumés comme suit :

1. La détérioration des conditions d'existence des catégories étudiées est liée à la baisse tant des salaires réels perçus par les ouvriers du secteur public industriel et les travailleurs $d u$ secteur tertiaire que du revenu des travailleurs du secteur informel que constituent les journaliers.

2. Le coût élevé de la reproduction des conditions d'existence des enquêtés et de leurs familles (dépenses alimentaires, d'habillement, d'enseignement, de santé, de logement et de loisirs) conduit à la réduction de la consommation d'un certain nombre de produits alimentaires (viande, poulet, poisson, fruits) dans les familles pauvres pour pouvoir couvrir les frais d'enseignement et de santé, et les loisirs.

3. Les enquêtés s'efforcent de réduire les difficultés de l'existence de diverses manières : par la constitution de familles nombreuses, le travail de la femme au sein du ménage et à l'extérieur, l'émigration, la pratique d'une seconde activité, et le recours aux dons de bienfaisance.

4. L'insuffisance du revenu, le coût élevé de la vie, l'endettement, les querelles entre les conjoints et enfin le logement sont les principaux problèmes dont souffrent les ménages. Sur un autre plan, la pauvreté, la détérioration des services et la pollution de l'environnement sont vus comme les principaux maux dont souffrent les quartiers où ils vivent. L'opinion des enquêtés sur le rôle du Conseil local est globalement négative.

À ce point de l'enquête, la conscience qu'ont les enquêtés de leur situation apparait spontanée et immédiate et ne relève en aucun cas d'une conscience de classe. 


\section{BIBLIOGRAPHIE}

AL-'AMRÛSI Salâh, 1992, « Mulâhadhât muwgaza hawla al-mas'ala al-fallâhiyya min mandhûr alishtirâkiyya al-'ilmiyya » (Brèves remarques sur la question paysanne du point de vue du socialisme scientifique), dans Al-Mas'ala al-fallâhiyya wa-l-zirâ'iyya fí Misr (La question des paysans et de l'agriculture en Égypte), colloque du Centre de recherches arabes, Le Caire.

FRIEDMAN L. A., 1989, L'évolution capitaliste en Égypte : la classe laborieuse égyptienne, traduit en arabe par Zuhdi AL-SHÂMÎ, Al-Tatawwur al-ra'smâlîfî Misr, Le Caire, Dâr al-'âlam al-gadîd.

AL-'ÎSAWÎ Ibrahim Hasan, 1989, Nahw kharîta tabaqiyya li-Misr, al-ishkâlât al-nadhariyya wa-l-iqtirâb al-manhagi min al-wâqi' al-tabaqî al-misrî (Problématique théorique et approche méthodologique de la réalité de classe en Égypte), Le Caire, Centre national de recherches sociales et criminologiques.

KISHK Hasanayn, 1995, 'Ummâl al-zirâ 'a fî Misr (1952-1995) : al-awdâ'al-iqtisâdiyya wa-l-igtimâ'iyya li-l-'ummâl al-zirâ'iyyîn al-ugarâ' (Les ouvriers agricoles en Égypte, 1952-1995 : les conditions économiques et sociales), Le Caire, al-Mahrûsa li-l-nashr.

\section{KORAYEM Karîma :}

- 1992, Poverty in Egypt, Literature Review (1985-1991), The Third World Forum and the Unicef, Cairo.

- 1994, Al-Faqr wa tawzî' al-dakhl fi Misr (Pauvreté et distribution du revenu en Egypte), Third World Forum, Middle East Office, Cairo.

ZAKî Ramzî :

- 1993a, « Anmât al-intâg wa-l-tawzî' wa-l-istihlâk al-sâ'ida fi-l-watan al-'arabî wa in'ikâsâtiha 'alâ awdâ' al-tanmiya al-bashariyya » (Les modes de production, de distribution et de consommation dominants dans le monde arabe et leurs répercussions sur les conditions du développement humain), communication présentée au colloque Al-tanmiya al-bashariyya fi-l-watan al-'arabî (Ledéveloppement humain dans le monde arabe), Commission économique et sociale pour l'Asie de l'Ouest, Ligue des États arabes, Programme des Nations unies pour le développement (PNUD), Le Caire, décembre.

-1993b, Al-librâliyya al-mustabidda (Le libéralisme despotique), Le Caire, Dâr Sîna.

\section{ANNEXES}

\section{Tableau 1 : Structure par âge de l'échantillon}

\begin{tabular}{|l|c|c|c|c|}
\hline Tranche d'âge & Hommes & Femmes & Total & $\%$ \\
\hline 20 à 29 & 17 & 8 & 25 & 37,3 \\
\hline 30 à 39 & 11 & 14 & 25 & 37,3 \\
\hline 40 à 49 & 10 & 2 & 12 & 17,9 \\
\hline 50 à 59 & 4 & 1 & 5 & 7,5 \\
\hline Total & 42 & 25 & 67 & 100 \\
\hline
\end{tabular}


Tableau 2 : Structure de l'échantillon selon le secteur d'activité

\begin{tabular}{|l|c|c|c|c|}
\hline Secteur d'activité & Hommes & Femmes & Total & $\%$ \\
\hline Secteur public industriel & 12 & 7 & 19 & 30 \\
\hline Secteur tertiaire public & 13 & 7 & 20 & 30 \\
\hline Secteur tertiaire privé & 7 & 2 & 9 & 13 \\
\hline Joumaliers & 6 & 6 & 12 & 17 \\
\hline Chômeurs & 4 & 3 & 7 & 10 \\
\hline Total & 42 & 25 & 67 & 100 \\
\hline
\end{tabular}

Tableau 3 : Structure de l'échantillon selon le niveau d'instruction

\begin{tabular}{|l|c|c|c|c|}
\hline Niveau d'instruction & Hommes & Femmes & Total & $\%$ \\
\hline Analphabètes & 15 & 10 & 25 & 37,4 \\
\hline Sait lire et écrire & 4 & - & 4 & 5,9 \\
\hline Certificat du primaire & 2 & 2 & 4 & 5,9 \\
\hline Certificat du préparatoire & 2 & 1 & 3 & 4,5 \\
\hline Certificat du secondaire & 12 & 12 & 24 & 35,9 \\
\hline Instituts techniques & 4 & - & 4 & 5,9 \\
\hline Formation professionnelle & 1 & - & 1 & 1,5 \\
\hline Diplômes universitaires & 2 & - & 2 & 3 \\
\hline Total & 42 & 25 & 67 & 100 \\
\hline
\end{tabular}

Tableau 4 : Structure de l'échantillon selon l'état matrimonial

\begin{tabular}{|l|c|c|c|c|}
\hline État matrimonial & Hommes & Femmes & Total & $\%$ \\
\hline Célibataire & 13 & 4 & 17 & 25,4 \\
\hline Marié & 29 & 18 & 47 & 70,2 \\
\hline Veuf & - & 2 & 2 & 2,9 \\
\hline Divorce & - & 1 & 1 & 1,5 \\
\hline Total & 42 & 25 & 67 & 100 \\
\hline
\end{tabular}

Tableau 5 : Nombre d'invidus par ménage (y compris l'enquêté) selon l'état matrimonial

\begin{tabular}{|c|c|c|c|c|c|c|}
\hline \multirow{2}{*}{$\begin{array}{l}\text { Nb d'individus } \\
\text { par ménage }\end{array}$} & \multicolumn{4}{|c|}{ État matrimonial } & \multirow[t]{2}{*}{ Total } & \multirow[t]{2}{*}{$\%$} \\
\hline & Célibataires & Mariés & Divorcés & Veufs & & \\
\hline 1 à 4 & 4 & 18 & 1 & 1 & 24 & 35,8 \\
\hline 5 à 7 & 12 & 23 & - & 1 & 36 & 53,7 \\
\hline 8 à 12 & 1 & 6 & . & . & 7 & 10,5 \\
\hline Total & 17 & 47 & 1 & 2 & 67 & 100 \\
\hline
\end{tabular}


Tableau 6 : Répartition des ménages de l'échantillon selon le niveau de revenu

\begin{tabular}{|c|c|c|c|c|c|c|}
\hline \multirow{2}{*}{$\begin{array}{l}\text { Revenus du } \\
\text { ménage (£e) }\end{array}$} & \multicolumn{4}{|c|}{ Secteur d'activité } & \multirow[t]{2}{*}{ Total } & \multirow[t]{2}{*}{$\%$} \\
\hline & $\begin{array}{l}\text { Secteur public } \\
\text { industriel }\end{array}$ & $\begin{array}{c}\text { Secteur tertiaire } \\
\text { public }\end{array}$ & $\begin{array}{c}\text { Secteur tertiaire } \\
\text { privé }\end{array}$ & $\begin{array}{l}\text { Journaliers et } \\
\text { chômeurs }\end{array}$ & & \\
\hline-200 & 4 & - & 3 & 10 & 17 & 25 \\
\hline 200 à 400 & 9 & 11 & 5 & 5 & 30 & 45 \\
\hline 400 à 600 & 5 & 5 & - & 2 & 12 & 18 \\
\hline 600 à 800 & 1 & 2 & 1 & - & 4 & 6 \\
\hline 800 à 1000 & - & 2 & - & - & 2 & 3 \\
\hline 1000 à 1200 & - & - & - & 2 & 2 & 3 \\
\hline Total & 19 & 20 & 9 & 19 & 67 & 100 \\
\hline
\end{tabular}

Tableau 7 : Répartition des ménages de l'échantillon selon la part des dépenses d'alimentation dans le revenu total

\begin{tabular}{|c|c|c|c|c|c|c|}
\hline \multirow{2}{*}{$\begin{array}{l}\% \text { des dépenses } \\
\text { alimentaires }\end{array}$} & \multicolumn{4}{|c|}{ Secteur d'activité } & \multirow[t]{2}{*}{ Total } & \multirow[t]{2}{*}{$\%$} \\
\hline & $\begin{array}{c}\text { Secteur public } \\
\text { industriel }\end{array}$ & $\begin{array}{l}\text { Secteur tertiaire } \\
\text { public }\end{array}$ & $\begin{array}{c}\text { Secteur tertiaire } \\
\text { privé }\end{array}$ & $\begin{array}{l}\text { Journaliers et } \\
\text { chômeurs }\end{array}$ & & \\
\hline-20 & . & - & 1 & 2 & 3 & 4 \\
\hline 20 à 40 & 3 & 3 & 2 & 2 & 10 & 15 \\
\hline 40 à 60 & 4 & 6 & 1 & 1 & 12 & 18 \\
\hline 60 à 80 & 6 & 3 & 1. & 3 & 13 & 20 \\
\hline 80 à 100 & 4 & 6 & 2 & 6 & 18 & 27 \\
\hline Non précisé & 3 & 4 & 1 & 3 & 11 & 16 \\
\hline Total & 17 & 18 & 7 & 14 & 67 & 100 \\
\hline
\end{tabular}


Tableau 8 : Scolarisation des enfants des enquêtés

\begin{tabular}{|c|c|c|c|c|c|c|}
\hline \multirow{2}{*}{$\begin{array}{c}\text { Scolarisation des } \\
\text { enfants }\end{array}$} & \multicolumn{4}{|c|}{ Secteur d'activité } & \multirow[t]{2}{*}{ Total } & \multirow[t]{2}{*}{$\%$} \\
\hline & $\begin{array}{c}\text { Secteur public } \\
\text { industriel }\end{array}$ & $\begin{array}{l}\text { Secteur tertiaire } \\
\text { public }\end{array}$ & $\begin{array}{l}\text { Secteur tertiaire } \\
\text { privé }\end{array}$ & $\begin{array}{l}\text { Journaliers et } \\
\text { chômeurs }\end{array}$ & & \\
\hline 1. Âge préscolaire & 14 & 18 & 5 & 9 & 46 & 22,1 \\
\hline $\begin{array}{l}\text { 2- Scolarisés } \\
\text { Cycle primaire } \\
\text { Cycle préparatoire } \\
\text { Cycle secondaire } \\
\text { Instituts techniques } \\
\text { Université } \\
\text { Total }\end{array}$ & $\begin{array}{c}16 \\
8 \\
8 \\
2 \\
1 \\
35\end{array}$ & $\begin{array}{c}19 \\
8 \\
7 \\
- \\
1 \\
35\end{array}$ & $\begin{array}{l}3 \\
4 \\
- \\
- \\
- \\
7\end{array}$ & $\begin{array}{l}17 \\
9 \\
4 \\
2 \\
2 \\
34\end{array}$ & $\begin{array}{c}55 \\
29 \\
19 \\
4 \\
4 \\
111\end{array}$ & 53,4 \\
\hline $\begin{array}{l}\text { 3. Diplômés } \\
\text { du primaire } \\
\text { du préparatoire } \\
\text { du secondaire et des } \\
\text { Instituts techniques } \\
\text { de l'Université } \\
\text { Total }\end{array}$ & $\begin{array}{l}1 \\
2 \\
1 \\
1 \\
4 \\
\end{array}$ & $\begin{array}{l}1 \\
1 \\
3 \\
5 \\
5\end{array}$ & $\begin{array}{l}\cdot \\
\cdot \\
6 \\
\cdot \\
6\end{array}$ & $\begin{array}{l}- \\
3 \\
1 \\
3 \\
7\end{array}$ & $\begin{array}{c}2 \\
6 \\
11 \\
3 \\
22 \\
\end{array}$ & 10,6 \\
\hline $\begin{array}{l}\text { 4- Abandon en cours } \\
\text { d'études } \\
\text { après le certificat } \\
\text { primaire } \\
\text { après le certificat } \\
\text { préparatoire } \\
\text { Total }\end{array}$ & . & $\begin{array}{l}1 \\
1 \\
\end{array}$ & $\begin{array}{l}1 \\
3 \\
\end{array}$ & $\begin{array}{c}8 \\
3 \\
11\end{array}$ & $\begin{array}{l}17 \\
5 \\
22 \\
\end{array}$ & 10,6 \\
\hline 5- Non-scolarisés & $\cdot$ &. & 1 & 6 & 7 & 3,4 \\
\hline Total & 60 & 59 & 22 & 67 & 208 & 100 \\
\hline
\end{tabular}

Tableau 9 : Répartition des ménages de l'échantillon selon la part des dépenses d'éducation dans le revenu total

\begin{tabular}{|c|c|c|c|c|c|c|}
\hline \multirow{2}{*}{$\begin{array}{l}\% \text { des } \\
\text { dépenses } \\
\text { d'éducation }\end{array}$} & \multicolumn{4}{|c|}{ Secteur d'activité } & \multirow[t]{2}{*}{ Total } & \multirow[t]{2}{*}{$\%$} \\
\hline & $\begin{array}{c}\text { Secteur public } \\
\text { industriel }\end{array}$ & $\begin{array}{c}\text { Secteur tertiaire } \\
\text { public }\end{array}$ & $\begin{array}{c}\text { Secteur tertiaire } \\
\text { privé }\end{array}$ & $\begin{array}{l}\text { Journaliers et } \\
\text { chômeurs }\end{array}$ & & \\
\hline - de 10 & 3 & 7 & 2 & 3 & 15 & 22,4 \\
\hline de 10 à 20 & 8 & 6 & . & 6 & 20 & 29,9 \\
\hline de 20 à 30 & 1 & 4 & - & 2 & 7 & 10,4 \\
\hline de 30 à 40 & $\cdot$ & - & . & 1 & 1 & 1,5 \\
\hline $40 \mathrm{et}+$ & 5 & 1 & 1 & 3 & 8 & 11,9 \\
\hline $\begin{array}{l}\text { Ménages non } \\
\text { concernés* }\end{array}$ & 5 & 2 & 6 & 3 & 16 & 23,9 \\
\hline Total & 19 & 20 & 9 & 19 & 67 & 100 \\
\hline
\end{tabular}

" Ménages ayant soit des enfants non encore scolarisés, soit des enfants ayant terminé ou abandonné leurs études. 
Tableau 10 : Répartition des ménages enquêtés se lon la part des dépenses de santé dans le revenu total

\begin{tabular}{|c|c|c|c|c|c|c|}
\hline \multirow[t]{2}{*}{$\%$} & \multicolumn{4}{|c|}{ Secteur d'activité } & \multirow[t]{2}{*}{ Total } & \multirow[t]{2}{*}{$\%$} \\
\hline & $\begin{array}{c}\text { Secteur public } \\
\text { industriel }\end{array}$ & $\begin{array}{c}\text { Secteur tertiaire } \\
\text { public }\end{array}$ & $\begin{array}{c}\text { Secteur tertiaire } \\
\text { privé }\end{array}$ & $\begin{array}{c}\text { Journaliers et } \\
\text { chômeurs }\end{array}$ & & \\
\hline .10 & 11 & 14 & 8 & 9 & 42 & 62,7 \\
\hline 10 à 20 & 3 & 2 & - & 9 & 14 & 20,9 \\
\hline 20 à 30 & 1 & - & 1 & - & 2 & 2,9 \\
\hline 30 à 40 & - & - & - & 1 & 1 & 1,5 \\
\hline+40 & 1 & 1 & - & $\therefore$ & 2 & 2,9 \\
\hline Non précisé & 3 & 3 & - & - & 6 & 8,9 \\
\hline Total & 19. & 20 & 9 & 19 & 67 & 100 \\
\hline
\end{tabular}

Tableau 11 : Type de logement

\begin{tabular}{|c|c|c|c|c|c|c|}
\hline \multirow{2}{*}{$\begin{array}{c}\text { Type de } \\
\text { logement }\end{array}$} & \multicolumn{4}{|c|}{ Secteur d'activité } & \multirow[t]{2}{*}{ Total } & \multirow[t]{2}{*}{$\%$} \\
\hline & $\begin{array}{l}\text { Secteur public } \\
\text { industriel }\end{array}$ & $\begin{array}{c}\text { Secteur tertiaire } \\
\text { public }\end{array}$ & Secteur privé & $\begin{array}{c}\text { Journaliers et } \\
\text { chômeurs }\end{array}$ & & \\
\hline Appartement loué & 9 & 4 & 2 & 8 & 23 & 34,4 \\
\hline $\begin{array}{l}\text { Chambre louée } \\
\text { avec sanitaires } \\
\text { communs }\end{array}$ & - & $\therefore$ & 2 & 2 & 4 & $5,9^{\circ}$ \\
\hline $\begin{array}{l}\text { Appartement loué } \\
\text { auprès de } \\
\text { l'employeur }\end{array}$ & 2 & 6 & - & 2 & 10 & 14,9 \\
\hline HLM & - & 1 & - & - & 1 & 1,6 \\
\hline $\begin{array}{l}\text { Maison de } \\
\text { location }\end{array}$ & - & . & 1 & 1 & 2 & 2,9 \\
\hline $\begin{array}{l}\text { Maison } \\
\text { appartenant au } \\
\text { père de l'enquêté }\end{array}$ & 4 & 6 & 3 & 3 & 16 & 23,9 \\
\hline $\begin{array}{l}\text { Maison } \\
\text { appartenant à } \\
\text { l'enquêté }\end{array}$ & 4 & 3 & - & 2 & 9 & 13,5 \\
\hline $\begin{array}{l}\text { Appartement } \\
\text { appartenant à } \\
\text { l'enquêté }\end{array}$ & - & - & 1 & 1 & 2 & 2,9 \\
\hline Total & 19 & 20 & 9 & 19 & 67 & 100 \\
\hline
\end{tabular}

\section{NOTES}

1. Dans une autre étude importante, Ramzi Zakî analyse le rôle de l'application du programme d'ajustement structurel dans la relance de l'accumulation capitaliste dans les pays du centre capitaliste, par le biais des mécanismes et des conditions nouvelles mises en place pour usurper les ressources des pays de la périphérie. Voir Zakî (1993b, p. 41-76).

2. Ce pourcentage renvoie à une ligne de pauvreté calculée sur la base de la distribution des revenus par ménage. Or, le taux donné pour 1981 -1982 est établi sur la base de la distribution des dépenses de consommation. Calculé sur la même base, le taux d'incidence de la pauvreté pour 1990-1991 est de $36 \%$. 
3. Les mesures de «libéralisation» de l'agriculture égyptienne entamées au milieu des années quatre-vingt ont conduit à une aggravation de la séparation entre les producteurs directs et les biens de production (en particulier la terre). Sur cette question, voir Kishk (1995, p. 19-22).

4. Niveau BEPC (N.D.E).

5. Toutes les sources de revenu du ménage ont été prises en compte, à l'exception de l'indemnité de repas dont ne bénéficient que les ouvriers du secteur public. Notons que certains enquêtés déclarent manifestement un revenu inférieur à la réalité.

6. Il est à noter que certains enquêtés font des déclarations à la baisse quand il s'agit du revenu du ménage, et à la hausse quand il s'agit des dépenses. La part des dépenses alimentaires et des dépenses d'enseignement et de santé déclarée tend à être majorée. La même observation revient dans de nombreuses études comme celles de Korayem (1994, p. 12 et note p. 13).

7. Il n'est pas précisé dans cette étude s'il s'agit d'une livre par jour ou par mois, la deuxième réponse étant la plus probable vu l'ironie avec laquelle il déclare ce montant ridicule.

8. Forme de solidarité informelle entre un groupe de personnes qui cotisent, pour une durée déterminée, à hauteur d'une certaine somme, chacune d'entre elles percevant, à tour de rôle, la totalité des cotisations mensuelles.

9. Magasin coopératif d'État où les vêtements sont vendus avec une carte de rationnement pour les familles à revenus limités.

10. Voir Kishk (1995, p. 97-100).

\section{INDEX}

Mots-clés : paupérisation, pauvreté 